\title{
Application and Development of Intelligent Medicine in Traditional Chinese Medicine*
}

\author{
Yu-yu DUAN ${ }^{1}$, Peng-ran LIU², Tong-tong $\mathrm{HUO}^{2}$, Song-xiang LIU², Song YE ${ }^{3 \#}$, Zhe-wei YE \\ ${ }^{1}$ Hubei University of Chinese Medicine, Wuhan 430072, China \\ ${ }^{2}$ Department of Orthopedics, Union Hospital, Tongji Medical College, Huazhong University of Science and Technology, Wuhan \\ 430022, China \\ ${ }^{3}$ Hubei Provincial Hospital of Traditional Chinese Medicine, Wuhan 430060, China
}

(C) Huazhong University of Science and Technology 2021

\begin{abstract}
[Abstract] As modern science and technology constantly progresses, the fields of artificial intelligence, mixed reality technology, remote technology, etc. have rapidly developed. Meanwhile, these technologies have been gradually applied to the medical field, leading to the development of intelligent medicine. What's more, intelligent medicine has greatly promoted the development of traditional Chinese medicine (TCM), causing huge changes in the diagnosis of TCM ailments, remote treatment, teaching, etc. Therefore, there are both opportunities and challenges for inheriting and developing TCM. Herein, the related research progress of intelligent medicine in the TCM in China and abroad over the years is analyzed, with the purpose of introducing the present application status of intelligent medicine in TCM and providing reference for the inheritance and development of TCM in a new era.
\end{abstract}

Key words: intelligent medicine; traditional Chinese medicine; development

As early as in the Qin and the Han Dynasties (221 $\mathrm{BC}$ to $\mathrm{AD} 220$ ), the Huang Di Nei Jing (Innner Canon of Yellow Emperor) marks when China's medical theoretical system began to take shape. In addition, the Shang Han Za Bing Lun (Treatise on Febrile Diseases and Miscellaneous Illnesses) establishes the general principle of treatment based on syndrome differentiation of traditional Chinese medicine (TCM). Syndrome differentiation treatment, the principle followed by TCM, is similar to the concept of precision medicine proposed by modern Western medicine, and both of these types of medicine reflect the philosophy of personalized medicine, indicating that TCM is forward-looking, satisfies the requirements of diagnosis and treatment in modern society, and has yielded good results in clinical applications and research $^{[1,2]}$. However, as TCM is impacted by modern intelligent medical technology, figuring out a way to effectively promote the inheritance and development of TCM as well as to realize efficient diagnosis and

Yu-yu DUAN, E-mail: 892275006@qq.com

\#Corresponding authors, Zhe-wei YE, E-mail: yezhewei@ hust.edu.cn; Song YE, E-mail: afk548@163.com

${ }^{*}$ This study was supported by grants from the National Natural Science Foundation of China (No. 81974355 and No. 82172525) and a Hubei Province Technology Innovation Major Special Project (No. 2018AAA067). treatment methods will make a difference. Hence, this paper summarizes the applications of intelligent medicine in the diagnosis, long-distance treatment, and teaching of TCM based on the review and analysis of its applications in TCM in China and abroad, aiming to provide fresh ideas for the combination of TCM and intelligent medicine as well as a reference for TCM inheritance and development in a new era.

\section{BACKGROUND OF INTELLIGENT MEDICINE DEVELOPMENT}

With the development of cutting-edge science and technology, the concept of intelligent medicine has been put forward. As an emerging interdiscipline of medicine, science, and engineering, it closely integrates medicine and a series of advanced technologies, including artificial intelligence (AI), mixed reality (MR), computer-assisted navigation in surgery, threedimensional (3D) printing, telemedicine, and medical big data. From 1950 to 1980 , intelligent medicine continued to improve and laid the foundation in the first phase of development. During the second phase of development in the next decade (1980-1990), the acquisition and construction of medical information updated intelligent medicine. The third phase of development occurred from 1990 to 2010, when intelligent medicine could provide objective decisions 
for diseases. With the development of cutting-edge science and technology, human medicine has welcomed the third era, namely the era of intelligent medicine (the first era is the era of empirical medicine, and the second one is the era of evidence-based medicine). Therefore, intelligent technologies have gradually become important medical auxiliary tools in the medical field and have greatly improved the learning and understanding abilities of humans in observing, touching, and listening. As a result, these technologies have a profound influence on medical treatment, including reducing doctors' work intensity and medical costs as well as improving the efficiency of diagnosis and treatment, safety, and patient satisfaction. More importantly, they have injected an impetus into medical reform and innovation as well as brought great transformation to healthcare, thus indicating that medicine is ushering in an era of precision, intelligence, and remoteness ${ }^{[3]}$. Moreover, the traditional medical model of TCM has been gradually transforming toward intelligent medicine with continuous strategies concerned with informatization and intelligence implemented in China as well as modern technologies applied in medicine. Therefore, the integration of new intelligent medical technologies with TCM is both an opportunity and a challenge for TCM development. Furthermore, their combination will certainly bring new models of inheritance and development.

\section{APPLICATION OF INTELLIGENT MEDICINE IN THE DIAGNOSIS OF AILMENTS BY TCM}

TCM sets great scores by treatment based on syndrome differentiation in the diagnosis and treatment of various conditions. Syndrome differentiation means identifying the syndrome type of a disease at the present stage by inspection, auscultation and olfaction, inquiry, and palpation in order to grasp the treatment principle. Meanwhile, giving treatment means defining the treatment approach in line with the differentiated syndrome and the treatment principle, including internal therapies and external ones like prescriptions, acupuncture, moxibustion, and massage ${ }^{[4]}$. Nowadays, TCM and modern medicine refer to diagnosis based on syndrome differentiation and disease differentiation, with the integration of modern medical technologies to make a diagnosis and treatment plan. Such a method helps to better understand a disease in different phases and to make full use of both TCM and Western medicine techniques. Therefore, both diseases and syndromes can be equally treated, and patients will get proper treatment. However, to some extent, there are still some disputes about four examinations used in treatments based on syndrome differentiation of TCM because the different syndrome information collected and diverse choices of the syndrome differentiation model made by physicians will bring about different results. Moreover, there are such problems like the conflict of failing to change a prescription, giving treatment based on syndrome differentiation, and the disappearance of symptoms and signs with uncured diseases, so some people question the objectivity of TCM. To some extent, the lack of objective indicators and patients' doubts will make it difficult to use TCM theories comprehensively and effectively in the diagnosis and treatment of various ailments. Consequently, the clinical efficacy will be affected, which will indirectly hinder the further exploration of $\mathrm{TCM}^{[5]}$. In contrast, the development of intelligent medicine has brought new possibilities to solve the abovementioned problems.

\subsection{Application of Intelligent Medicine in Tongue Inspection}

Tongue inspection means observing the texture and coating of the tongue, including the color, shape, and dynamic state. What's more, TCM holds that different areas of the tongue correspond to different Zang and $\mathrm{Fu}$ organs; therefore, tongue inspection serves as one of the most valuable methods in clinical diagnosis ${ }^{[6]}$. However, it is difficult to provide relatively objective diagnostic results, since the traditional diagnostic method is comparatively subjective and will be influenced by light and personal factors.

In the early 1980 s, there were relevant studies about tongue inspection in China and abroad. Technologies like the tongue image segmentation algorithm (threshold method and Markov random fields model) have basically extracted and classified tongue information. The further development of image extraction technology and mass storage technology has enabled a clearer extraction for tongue imaging and established a large-sample database of tongue images, laying a foundation for the complete reproduction of tongue images and machine learning in the future.

Although the intelligent medical technologies mentioned above have preliminarily realized feature extraction and recognition of tongue images, the twodimensional (2D) images still fail to offer complete information in detail. As a result, there is a lack of key information regarding tongue images, such as the shape and thickness of the tongue. Continuous efforts are being made to tackle these problems. Researchers like Kanawong et $a l^{[7]}$ have improved the existing system of tongue image analysis and designed a new algorithm based on color and spatial features, which has been applied to extract objective features, especially the petechia distributed on the tongue surface. Next, 3D ultrasonic imaging technology will soon be designed and become the key technology to study tongue images in China and abroad. Depending on such a technology, more complete tongue information, such as the contour, the thickness of the tongue coating, and 
the tongue flexibility, can be extracted ${ }^{[8]}$. Some studies have established a tongue simulation system with a network model. The 3D modeling of the tongue with magnetic resonance as its basis has gradually begun to utilize $3 \mathrm{D}$ magnetic resonance imaging technology for $3 \mathrm{D}$ reconstruction, realizing the simulation of the real dynamic state of the tongue ${ }^{[9,10]}$. Finally, these technologies will be applied to the objective extraction of the tongue image clinically, and the intelligent system of tongue image analysis will provide assistance to clinicians.

Intelligent medicine helps to solve the problem of insufficient information for reconstruction of tongue images, and many studies have achieved disease prediction with intelligent extraction of tongue images by using intelligent recognition technology, based on the tongue image extracted by the full-featured multiinformation system. For example, Wang et al ${ }^{[11]}$ have statistically analyzed the distribution and characteristics of tongue color in 106 patients with diabetes mellitus type 2 by using a tongue image instrument to collect tongue image pictures. As a result, they found that the course of diabetes and relevant biochemical indexes are associated with changes of the tongue color. In addition, it has been concluded that changes of the tongue color are important in TCM diagnosis to evaluate the glycemic control of patients as well as to predict the microvascular complications of the heart, brain, and kidney. Moreover, Li et al ${ }^{[12]}$ have statistically analyzed 214 patients using a TCM tongue and face instrument. After extraction of the tongue image features and intelligent discrimination, they concluded that the changes in the parameters of tongue and face images of patients with H-type hypertension are consistent with the pathogenesis of Yang hyperactivity and dampness exuberance of hypertension. Therefore, the prediction and early prevention of hypertension can be realized according to the results of tongue and face inspection. Furthermore, Kanawong et $a l^{[7]}$ used the assistance of a tongue image system to extract the image features, achieved machine learning with chronic gastritis as examples for classification and extraction, and established the relationship between features and syndrome types through the examples, thus verifying the objective association between tongue images and syndromes. In this way, such combinations of intelligent medical technologies and TCM serve as effective tools for comprehensive collection of tongue images and disease prediction.

\subsection{Application of Intelligent Medicine in Pulse Diagnosis}

Pulse differentiation in TCM is relatively subjective and obscure; therefore, diverse results are often obtained due to different physicians and methods. With the progress of modern technology, researchers have gradually transformed the signals from the human body into more intuitive visual images. Additionally, the expressions have also been changed from simple "waves" to today's complete "diagrams", showing that more and more advanced imaging methods are being combined. A pulse diagnostic instrument has emerged, which provides new possibilities for objective pulse diagnosis obtained by TCM. Such an instrument needs two key technologies: sensor technology and pulse recognition technology. In terms of the former, a pressure sensor is the main research direction, ranging from the previous single-head sensor to a three-head sensor, and then to the humanized flexible pressure sensor of pulse. In terms of pulse recognition technology, the pulse spectrum is mainly used for human signal analysis, and the combination of the two can offer more reliable and objective data ${ }^{[13]}$.

In the 1860 s, the first sphygmograph was invented based on the lever principle of a spring. Based on the sensing wave, it was designed to collect a pulse wave at three different pulse-taking pressures: light, medium, and heavy. It evolved from simply recoding the sensing pressure of a single rigid surface to the positions of Cun, Guan, and Chi (three parts around the wrist). Although it is a recording mode that can simulate the movement intensity of various fingers, it still fails to exactly obtain the same features as those from pulse diagnosis through finger pressure. However, with the development of bionics and other related technologies, the optimized design of the acquisition point and the emerging ways of composite acquisition provide a new way for a pulse diagnostic instrument to collect objective pulse information. Lin et al ${ }^{[14]}$ have designed a method and equipment that can accurately measure the pulse waves, effectively solving the positioning issue during pulse diagnosis by accurately measuring the pulse information at Cun, Guan, and Chi and the features of light, medium, and heavy pulses. Similarly, Zhang et $a l^{[15]}$ have designed a composite pulse acquisition equipment, which is composed of a photoelectric sensor and a pressure sensor, and it can simultaneously collect the pulse information at Cun, Guan, and Chi for more pulse signals. Moreover, Jiang et $a l^{[16]}$ have designed a flexible resistance-type pressure sensor composed of polymer nanofibers and graphene. It has a higher sensitivity and sensing capability, and it can collect more detailed pulse information.

Furthermore, the pulse diagnostic instrument has been increasingly applied in clinical research. He et $a l^{[17]}$ have studied the relationship between constitution and pulse diagnosis. By comparing the pulse diagnosis information of the qi-depression constitution and mild constitution, they have found that the waves on the pulse diagnosis instrument vary dramatically, so they developed a technology that can apply wave data to clinical constitution analysis. In addition, Ding et $a l^{[18]}$ have analyzed nine types of pulses of spleen and 
stomach diseases using a pulse diagnostic instrument. As a result, they found that the equipment can accurately and effectively detect and distinguish nine types of pulses, reflecting the differences of waves and parameter changes of different pulses in an objective manner. Moreover, their research findings are likely to assist TCM telemedicine ${ }^{[19]}$. Kou et al ${ }^{[20]}$ have investigated the relationship between hemodynamics and a string-tight pulse, virtual pulse, and flat pulse with a smart phone-based wristband inflatable smart pulse instrument. They concluded that the instrument can distinguish the above three pulses and that the hemodynamic results detected by each group also have physiological and pathological significance.

With the continuous development of intelligent medical technology, pulse diagnosis using TCM is no longer hampered by space barriers. Based on the collection and transmission of pulse signals and the objective remote reproduction of the real pulse of patients, a new model of pulse diagnosis has taken shape, which injects a new driving force into the development of TCM pulse diagnosis.

\subsection{Application of an Eye Diagnostic Instrument in the Inspection}

As the saying goes that "The essence and Qi of the five Zang and six Fu organs are all reflected through the eyes," eye diagnosis is a characteristic of TCM. Diseases can be diagnosed through the changes of the eyes, which can reflect the changes of the five Zang and six Fu organs, providing important references for diseases diagnosis. Based on the five-wheel theory of TCM, Zhang et al have divided the eye into five parts based on the five orbiculus theory of TCM, namely the flesh orbiculus, blood orbiculus, $Q i$ orbiculus, wind orbiculus, and water orbiculus, corresponding to the eyelid, canthus, white part of the eye, eyeball, and pupil, which should be respectively and internally associated with the spleen, heart, lung, liver, and kidney. In this way, the equipment can analyze the information collected, and it is mainly divided into an information collector and a processor. Additionally, it is applied in the clinic by collecting eye images of patients with liver cirrhosis and healthy people for local binary patterns (LBP) analysis. The feature frequency of each image can have 255 layers, which refines the texture of eye images. Thus, a significant difference can be observed through the comparison of two LBP ring diagrams with feature frequency ${ }^{[21]}$.

In addition to the tongue diagnostic, pulse diagnostic, and eye diagnostic instruments introduced above, some researchers have also developed an integrated type of auxiliary diagnostic equipment. Due to the complex design, this kind of equipment can be represented by the BD-SZ Portable Four Diagnostic and Auxiliary Diagnostic Instrument developed and manufactured by Beijing University of Traditional
Chinese Medicine for its relative mature and good quality. This instrument can completely cover the information from the perspective of four diagnoses, and it is valuable for both clinical applications and teaching activities. It is composed of a pulse diagnostic instrument, tongue diagnostic instrument, smelling and inquiring diagnostic instruments, portable computer, four diagnostic and auxiliary diagnostic instrument software system, and portable output units. A clinical trial and evaluation have collected four types of diagnostic information from 30 subjects and provided intervention suggestions accordingly. Compared with the manual discrimination group, it was concluded that the instrument provides good repeatability, safety, and stability characteristics ${ }^{[22]}$.

\section{APPLICATION OF INTELLIGENT MEDICINE IN OTHER FIELDS OF TCM}

\subsection{Intelligent Syndrome Differentiation System through Machine Learning}

Treatment based on syndrome differentiation has always been the core of TCM diagnosis. Different extractions of symptom information by doctors and their individual choice of syndrome differentiation models will provide different results of syndrome differentiation. Eight-principle Syndrome Differentiation is the guiding principle of syndrome differentiation. Besides, there are other systems ranging from Six Meridian Syndrome Differentiation, DefenseQi-Nutrient-Blood Syndrome Differentiation, Triple Energizer Syndrome Differentiation, and Qi-BloodFluid Syndrome Differentiation. In addition, different syndrome differentiation models directly determine the accuracy and efficiency of clinical syndrome differentiation. The research and development of syndrome differentiation systems mainly consists of four parts: collection, review and search of medical records, statistical analysis, and visualization. Therefore, the way to optimize each algorithm will affect the accuracy and effectiveness of the syndrome differentiation system in $\mathrm{TCM}^{[23]}$.

As early as in 1987, Chen et al ${ }^{[24]}$ designed a diagnostic system providing consultations by numerous TCM experts to diagnose and treat stomachache and abdominal pain, based on AI and database technology. Moreover, a multi-level inference network model was established with experts as the main body to obtain complete diagnosis and treatment data. With the establishment and continuous renewal of the knowledge base, real-time information was made accessible to learners so that they could acquire and learn it both conveniently and efficiently. In the syndrome differentiation system of TCM, most algorithms are used as models to solve syndrome diagnosis or to design the model of the surface structure; these 
algorithms lack human subjective judgment, that is, the cognitive perspective from our brain. In this regard, Guo et $a l^{[25]}$ have found that there is a complex nonlinear relationship between symptoms (signs) and syndromes in clinical practice. They adopted such methods like Chainer and multi-label learning to establish a TCM diagnosis model of the syndrome for chronic gastritis. Furthermore, it has been proved that such a computer deep learning algorithm can effectively improve the accuracy of symptom recognition, thus establishing a syndrome diagnosis model to guide clinical practice. Additionally, Cao et a ${ }^{[26]}$ have established an intelligent syndrome differentiation model of gastroesophageal reflux disease based on machine learning and summarized that the intelligent model provides satisfactory diagnosis and disease prediction abilities that can be feasibly applied. Obviously, the syndrome differentiation system combined with intelligent medical technology affords effective assistance for the objective diagnosis and treatment by TCM. Likewise, Yang et $a{ }^{[27]}$ have designed an intelligent instrument to collect the information from the fundus image, tongue diagnosis, and pulse diagnosis for the early diagnosis of diabetes through the random forest algorithm of machine learning. This instrument can noninvasively and rapidly diagnose type 1 and type 2 diabetes. Its emergence not only improves the adverse prognosis of patients with diabetes whose diagnoses are limited by time and space, but it also provides medical support in remote mountainous areas. In this way, the syndrome differentiation system combined with intelligent medical technology can provide effective assistance for the objective diagnosis and treatment of ailments by TCM.

\subsection{Diagnosis and Treatment of Ailments by TCM through Telemedicine}

Telemedicine is an emerging medical service model whose advantages of accessibility, economic benefits, and social benefits break the traditional medical pattern and advance the global medical cause. It mainly depends on the design of platform software and communication network services, which determine its successful application. China enjoys a vast territory but with an uneven distribution of medical resources. Compared with coastal developed areas, other areas in China, especially the remote mountainous and border areas, lack high-quality medical and teaching resources. But with the assistance of telemedicine systems, the problem of unevenly distributed medical resources can be alleviated to a great extent ${ }^{[28,29]}$. Furthermore, the advent of the $5 \mathrm{G}$ era heralds that there will be more stable communication support to realize seamless connection between doctors and patients, even from a long distance.

The accurate intelligent analysis and remote transmission of TCM pulse images are realized on the basis of a long-distance intelligent pulse diagnostic system through a mobile phone and the mobile platform called Family Doctors. And with the simultaneous upload of tongue images and medical history data, patients are able to receive such services without leaving home as a remote consultation, further consultation, prescription, home delivery of drug, or taking the medicine nearby. Such remote services break the space limitations of traditional diagnosis and treatment ${ }^{[30]}$. During the outbreak of COVID-19 in 2020, numerous hospitals took advantage of remote technology and remote diagnosis platforms to provide group consultation, teaching, and case discussion. In this manner, a remote consultation model was established for TCM and Western medicine in combination to offer guidance for patients from a long distance, and satisfactory results have been achieved. What's more, these remote technologies made it possible to provide China's experience to the international community ${ }^{[31,32]}$. Overall, such a new model helps to diagnose and treat ailments by TCM as well as to improve the recognition and acceptability of TCM.

\subsection{TCM Education Based on AI Technology}

Cultivating TCM professionals to solve the problem of a shortage of talent has always been the core issue for the inheritance and development of TCM. It has been admitted that traditional masterapprenticeship training is an important and effective means for the inheritance and development of TCM. At the same time, it is also an essential step in line with the regular pattern of TCM talent training. Education in modern universities can make up for the defects of standardized master-apprenticeship training and single clinical thought, but it cannot replace the contributions made by such a secession pattern in the cultivation of TCM talents ${ }^{[33]}$. Therefore, determining ways to combine the inherent advantages and to reasonably apply modern teaching methods are important issues worthy of consideration for the development and inheritance of TCM.

As branches of intelligent medicine, virtual reality (VR) and AI represent the cutting-edge ones of innovative technology in the field of information technology. VR technology is a computer simulation system that can create a virtual world that one can experience. It generates a simulated environment relying on a computer, and it combines interactive 3D dynamic scenes using information from multiple sources with entity behaviors so that users can immerse themselves in the fictional environment. The system is characterized by perception, interaction, and subjectivity ${ }^{[34]}$. Based on computer algorithms, AI is a type of intelligent activity that studies human brain intelligence in order to simulate, develop, and extend people's intelligence as well as to finally realize a computer autonomic respons $\mathrm{e}^{[35,36]}$. For example, during 
the teaching process of meridian acupuncture, Rao et $a l^{[37]}$ have established and applied a virtual acupuncture system. This system can present 2D acupoints and circulation images by means of 3D simulation with VR technology, showing complex images of acupoints and circulations in a 3D manner. Therefore, learners can drag and spin at will to establish a convenient, independent, and omni-directional learning pattern. At the same time, the system also realizes the multimodal switching of the learning environment so that students can effectively master clinical regularity and application skills. Moreover, the learning process becomes more interesting so that the students' learning efficiency is improved. In addition, the technology of ancient classic exploration, based on AI technology, can sort out numerous TCM classics and famous medical records as well as explore the medication laws of TCM experts to realize intelligent compatibility of medicines. In this way, the most real teaching scenarios and materials are accessible to students. Scholars can acquire more effective learning materials and personal experience, which benefit their in-depth study. Such intelligent medical technologies have brought a new model to TCM education ${ }^{[38]}$.

\section{SUMMARY AND PROSPECT}

The integration of modern science and technology is inevitable for the effective inheritance and development of TCM; therefore, it is necessary to effectively integrate and make use of TCM medical and health resources as well as to improve its accessibility for health services. This is not only what TCM needs to take seriously in modern times, but it also offers opportunities and challenges posed by the times. Thus, the application of intelligent medicine in TCM will help to improve the objectification of TCM and greatly save medical and social resources, bringing considerable economic and social benefits to the development of $\mathrm{TCM}^{[39]}$.

It has been proved that it is worthy and meaningful to combine intelligent medicine and TCM as seen by the wide usage of intelligent medicine in TCM diagnosis, telemedicine, teaching, and other fields. Such a combination also brings good application prospects and breakthrough progress to this field. However, there are still some concerns about the combination at this stage: The first one is the lack of massive data accumulation and technological innovation; the second one is the lack of a perfect evaluation system; the third one is the lack of specific diagnostic application standards; the fourth one is slow development and low acceptance; the last one is the great talent gap of medical engineering professionals. All of the above problems restrict further integration and development of intelligent medicine and TCM.
Nevertheless, the authors believe that these problems will be addressed one by one as intelligent medical technologies are gradually improved. Intelligent medicine will eventually lead TCM to a bright future that meets the needs of the times. It is believed that intelligent medicine will become the major driver to improve medical quality and services of TCM with the rapid development of various technologies ${ }^{[40]}$.

\section{Conflict of Interest Statement}

The authors declare that there are no conflicts of interest relevant to this article.

\section{REFERENCES}

1 Alice YL, Guan B, Chen S, et al. Artificial intelligence meets traditional Chinese medicine: a bridge to opening the magic box of sphygmopalpation for pulse pattern recognition. Digit Chin Med (Chinese), 2021,4(1):1-8

2 Williams JR, Lorenzo D, Salerno J, et al. Current applications of precision medicine: a bibliometric analysis. Per Med, 2019,16(4):35-359

3 Tang PF, Zhang H, Li JT, et al. Surgery 4.0: The advent of the era of digital and intelligent surgical empowerment. Chin J Orthop Trauma (Chinese), 2019,21(3):185-188

4 Sun YN, Wan Y, Zhang Y, et al. Exploration study on TCM related specific outcome sets for evaluating effect of syndromes differentiation and treatment. Chin J Tradit Chin Med Pharm (Chinese), 2020,35(10):48154819

5 Dang Y, Shi JP, Lan DD, et al. Comparative study of syndrome differentiation and precision medicine under the combination of disease and syndrome. Chin J Tradit Chin Med Pharm (Chinese), 2019,34(12):5615-5617

6 Li X, Chen H, Qi X, et al. H-DenseUNet: Hybrid Densely Connected UNet for Liver and Liver Tumor Segmentation from CT Volumes. IEEE Trans Med Imaging, 2017,37(12):2663-2674

7 Kanawong R, Obafemi-Ajayi T, Liu D, et al. Tongue Image Analysis and Its Mobile App Development for Health Diagnosis. Adv Exp Med Biol, 2017,1005:99121

8 Naga Karthik EMV, Karimi E, Lulich SM, et al. Automatic tongue surface extraction from threedimensional ultrasound vocal tract images. J Acoust Soc Am, 2020,147(3):1623

9 Chen J, Luo C, Yu J, et al. Modeling a realistic 3D physiological tongue for visual speech synthesis. IEEE ICMEW, 2014.

10 Liu Z, Wang H, Xu H, et al. 3D Tongue Reconstruction Based on Multi-view Images and Finite Element. Adv Inf Sci Serv Sci, 2011,3(11):60-66

11 Wang CG, Shi XG, Cai YH, et al. Analysis of the characteristics distribution of tongue color and its related factors in 106 patients with type 2 diabetes. Mod J Integr Tradit Chin West Med (Chinese), 2020,29(20):21842187

12 Li YY, Zhao QQ, Wang YQ, et al. Analysis of color characteristic parameters of tongue and face image in patients with H-type hypertension. Mod Tradit Chin Med Mat Med-World Sci Technol (Chinese), 2020, 22(05):1581-1586 
13 Jiang Y, Liu CY, Zhang YD, et al. Research progress and new ideas of pulse taking instrument. Chin J Tradit Chin Med Pharm (Chinese), 2017,32(01):218-221

14 Lin FJ, Wu JK. Sensor array device for measuring pulse wave. CN: 109602399A. 2019-04-12

15 Zhang DP, Jiang ZX, Lu GM. Pulse collecting method and device. CN: 105962910A. 2016-09-28

16 Jiang K, Chen ZJ, Shen GZ, et al. Research and manufacture of a flexible resistance pressure sensor for human pulse detection. CN: 105708425A. 2016-06-29

17 He SM, Xie MZ. Research of the characteristics of pulse diagnosis information of Qi-depression and peaceful quality healthy population. Clin J Tradit Chin Med (Chinese), 2019,31(09):1686-1689

18 Ding S. Analysis and formation principle of nine pulse diagrams of spleen and stomach diseases detected by mobile pulse-taking inflatable instrument. Liaoning Univ Tradit Chin Med (Chinese), 2020.

19 Yang PY, Teng J, Qi XH. Research progress of modern pulse diagnostic instrument. Hunan J Tradit Chin Med (Chinese), 2018,34(4):202-204

20 Kou Y, Gao Q, Gao WY, et al. Relation between hemodynamics and 3 kings of pulse presentations by wristlet gas-filled type pulse diagnosis meter on intelligence handset. Clin J Med Off (Chinese), 2020, 48(09):1015-1016+1020

21 Zhang H, Zhao R, Hang LB, et al. Inspection device and information processing method for eye diagnosis of traditional Chinese medicine. CN: 111667905A. 202009-15

22 Niu TL, Yang XZ, Zhu QW, et al. Clinical trial and assessment of a portable apparatus for auxiliary diagnosis and treatment based on four TCM diagnostic methods. J Beijing Univ Tradit Chin Med (Chinese), 2011,34(04):231-235+289

23 Wei CF, Luo LQ, Yan JF. Research on construction of intelligent acquisition and analysis system of supporting medical cases for traditional Chinese medicine digital syndrome differentiation. J Hunan Univ Chin Med (Chinese), 2020,40(01):70-74

24 Chen P, Li J, Liang FL, et al. Study on versatile intelligence system of traditional Chinese medicine. J Luzhou Med Coll (Chinese), 1987(04):273-278

25 Liu GP, Yan JJ, Wang YQ, et al. Deep Learning Based Syndrome Diagnosis of Chronic Gastritis. Comput Math Methods Med, 2014(3-5):938350

26 Cao Y, Lu Y, Chen JX. Establishment of TCM intelligent pattern identification model of gastroesophageal reflux disease based on machine learning. J Beijing Univ Tradit Chin Med (Chinese), 2019,42(10):869-874

27 Yang X, Lai SJ, Chang HF, et al. Artificial IntelligenceBased Diagnosis of Diabetes Mellitus: Combining Fundus Photography with Traditional Chinese Medicine
Diagnostic Methodology. Biomed Res Int, 2021,2021: 5556057

28 Waqas A, Teoh SH, Lapo LV, et al. Harnessing telemedicine for provision of healthcare: Identifying research themes, hotspots and landmark theories. J Med Internet Res, 2020,22(10):e18835

29 Liu SX, Xie M, Ye ZW. Combating COVID-19-How Can AR Telemedicine Help Doctors More Effectively Implement Clinical Work. J Med Syst, 2020,44(9):141

30 Yang GY, Lei CH, Li YT, et al. Demonstration of remote TCM pulse diagnosis system and family doctor platform based on smart phones. Chin Gen Pract (Chinese), 2019,22(33):4128-4132

31 Chen X, Huang W, Liu BY, et al. Moxibustion therapy in prevention and treatment of coronavirus disease 2019 (COVID-19): construction and application of noncontact diagnosis and treatment mode. Zhong Guo Zhen Jiu (Chinese), 2020,40(10):1027-1033

32 Xie Y, Zhang JY, Wang HL, et al. Applications of blockchain in the medical field: A narrative review. J Med Internet Res, 2021,23(10):e28613

33 Sun Di, Guo LM, Tai DM. Development of TCM international education in the new era: logic, dilemma and path. Med Educ Res Pract (Chinese), 2021,29(01):1$3+9$

34 Portelli M, Bianco SF, Bezzina T, et al. Virtual reality training compared with apprenticeship training in laparoscopic surgery: a meta-analysis. Ann R Coll Surg Engl, 2020,102(9):672-684

35 Liu PR, Lu L, Huo TT, et al. Application progress of artificial intelligence technology in orthopedics. Chin J Orthop (Chinese), 2020,40(24):1699-1704

36 Liu SX, Xie M, Zhang ZC. A 3D hologram with mixed reality techniques for better understanding the pulmonary lesions of COVID-19: Randomized Controlled Trial. J Med Internet Res, 2021,23(9):e24081

37 Rao XD, Yu HB, Wu JH, et al. Practical experience of virtual acupuncture and moxibustion teaching D, system in the operation teaching course of Acupuncture Sciences. Chin Acupunct Moxibustion (Chinese), 2020, 40(08):877-879

38 Liao J, Wang JC, Yu Y, et al. Application of Artificial intelligence in the inheritance of famous TCM expert. Chin J Tradit Chin Med Parm (Chinese), 2020,35(04): 1671-1674

39 Alice YL, Guan B, Chen S, et al. Artificial intelligence meets traditional Chinese medicine: a bridge to opening the magic box of sphygmopalpation for pulse pattern recognition. Digit Chin Med, 2021,4(1):1-8

40 Wang Y, Shi X, Li L, et al. The Impact of Artificial Intelligence on Traditional Chinese Medicine. Am J Chin Med, 2021,49(6):1297-1314

(Received Oct. 4, 2021; Oct. 26, 2021) 